\title{
X-RAY PROPERTIES OF THE YOUNGEST RADIO SOURCES AND THEIR ENVIRONMENTS
}

\author{
Aneta Siemiginowska ${ }^{1}$, Matgosia Sobolewska ${ }^{1,2}$, Givlia Migliori ${ }^{3}$, Matteo Guainazzi $^{4,5}$, Martin Hardcastle ${ }^{6}$, \\ LuISA OSTORERo $^{7}$, AND ŁUKASZ STAWARz ${ }^{8}$ \\ ${ }^{1}$ Harvard Smithsonian Center for Astrophysics, 60 Garden St, Cambridge, MA 02138, USA; asiemiginowska@cfa.harvard.edu \\ ${ }^{2}$ Nicolaus Copernicus Astronomical Center, Bartycka 18, 00-716 Warsaw, Poland \\ ${ }^{3}$ Lab. AIM/Univ. Paris Diderot Paris 7/CNRS/CEA-Saclay, France \\ ${ }_{5}^{4}$ Institute of Space and Astronautical Science, JAXA, 3-1-1 Yoshinodai, Sagamihara, Kanagawa, 252-5210, Japan \\ ${ }^{5}$ European Space Astronomy Centre of ESA, P.O. Box 78, Villanueva de la Cañada, E-28691 Madrid, Spain \\ ${ }^{6}$ School of Physics, Astronomy and Mathematics, University of Hertfordshire, College Lane, Hatfield AL10 9AB, UK \\ ${ }^{7}$ Dipartimento di Fisica-Università degli Studi di Torino and Istituto Nazionale di Fisica Nucleare (INFN), Via P. Giuria 1, I-10125 Torino, Italy \\ ${ }^{8}$ Astronomical Observatory, Jagellonian University, ul. Orla 171, 30-244 Kraków, Poland \\ Received 2015 September 24; accepted 2016 March 10; published 2016 May 23
}

\begin{abstract}
We present the first results from our X-ray study of young radio sources classified as compact symmetric objects (CSOs). Using the Chandra X-ray Observatory we observed six CSOs for the first time in X-rays, and re-observed four CSOs already observed with XMM-Newton or BeppoSAX. We also included six other CSOs with archival data to built a pilot study of a sample of the 16 CSO sources observed in X-rays to date. All the sources are nearby, $z<1$, and the age of their radio structures $(<3000 \mathrm{yr})$ has been derived from the expansion velocity of their hot spots. Our results show the heterogeneous nature of the CSOs' X-ray emission, indicating a complex environment associated with young radio sources. The sample covers a range in X-ray luminosity, $L_{2-10 \mathrm{keV}} \sim 10^{41}$ $10^{45} \mathrm{erg} \mathrm{s}^{-1}$, and intrinsic absorbing column density of $N_{\mathrm{H}} \simeq 10^{21}-10^{22} \mathrm{~cm}^{-2}$. In particular, we detected extended $\mathrm{X}$-ray emission in 1718-649; a hard photon index of $\Gamma \simeq 1$ in $2021+614$ and $1511+0518$ consistent with either a Compton-thick absorber or non-thermal emission from compact radio lobes, and in $0710+439$ an ionized iron emission line at $E_{\text {rest }}=(6.62 \pm 0.04) \mathrm{keV}$ and $\mathrm{EW} \sim 0.15-1.4 \mathrm{keV}$, and a decrease by an order of magnitude in the $2-10 \mathrm{keV}$ flux since the 2008 XMM-Newton observation in $1607+26$. We conclude that our pilot study of CSOs provides a variety of exceptional diagnostics and highlights the importance of deep X-ray observations of large samples of young sources. This is necessary in order to constrain theoretical models for the earliest stage of radio source evolution and to study the interactions of young radio sources with the interstellar environment of their host galaxies.
\end{abstract}

Key words: galaxies: active - galaxies: jets - X-rays: galaxies

\section{INTRODUCTION}

Theory and simulations predict that radio sources are most luminous when they start growing within the very central regions of their host galaxies (Scheuer 1974; Begelman \& Cioffi 1989; Begelman 1996; Readhead et al. 1996a; Heinz et al. 1998). During this initial expansion a radio source is expected to interact strongly with the interstellar medium (ISM), inducing shocks, accelerating particles, heating the ISM, and accelerating gas to form an outflow (Begelman et al. 1984; Begelman \& Cioffi 1989; Bicknell et al. 1997; Silk \& Rees 1998; Reynolds et al. 2001). Empirical evidence for such interactions comes from observations of galactic-scale X-ray structures in nearby radio galaxies (e.g., Kraft et al. 2000; Mingo et al. 2011; Hardcastle et al. 2012; Siemiginowska et al. 2012), as well as from correlations between velocities of optical line-emitting gas and the morphology of radio outflows in parsec-scale radio sources (Holt et al. 2003; Morganti et al. 2003, 2013; Tadhunter et al. 2014). Such interactions, leading to energy exchange between a radio source and the ISM, are thought to contribute to feedback that governs the evolution of galaxies over cosmological timescales (e.g., Best et al. 2006; Croton et al. 2006). At present, though, we have only been able to study such interactions in relatively old $\left(>10^{4}-10^{5} \mathrm{yr}\right)$ large radio sources, while studies of young compact systems have been limited. Although the youngest sources would only impact the very central regions of their galaxy, they can strongly influence the sites directly responsible for the fuel supply to the active galactic nucleus (AGN), e.g., by heating and accelerating material away from the central regions (e.g., Bicknell et al. 1997; Holt et al. 2008; Wagner \& Bicknell 2011). Thus, these youngest sources could be critical to our understanding of the feedback processes impacting galaxy evolution.

The existing velocity measurements of radio hot spots and spectral aging studies indicate that compact symmetric objects (CSOs), a subclass of gigahertz-peaked spectrum (GPS) sources, are among the youngest extragalactic radio sources, with ages between $\sim 100$ and $3000 \mathrm{yr}$ (Owsianik \& Conway 1998; Taylor et al. 2000; An et al. 2012). Interestingly, there seems to be an excess of CSOs younger than $\sim 500 \mathrm{yr}$ (Gugliucci et al. 2005). This excess, together with a large number of compact radio sources $(<1 \mathrm{kpc})$ in comparison to large radio galaxies (O’Dea \& Baum 1997), may suggest that the radio jet activity is intermittent (Reynolds \& Begelman 1997; Czerny et al. 2009; Shulevski et al. 2012) or that the compact sources are short-lived (Readhead et al. 1996b; Kunert-Bajraszewska et al. 2010, 2015). In either case the youngest CSOs expand within the central $(\approx 100 \mathrm{pc})$ regions of the galaxies and contribute to the feedback.

The young age of the CSO sources has been challenged by a frustrated jet scenario in which the presence of a dense medium forces the jets to decelerate rapidly and prevents them from propagating beyond the central parsec-scale regions (van Breugel et al. 1984; Gopal-Krishna \& Wiita 1991; De Young 1993, 1997; Carvalho 1994; Perucho 2015). This 
would introduce a systematic bias to the kinematic age measurements. However, until now there has been no evidence for a dense enough medium to halt the jets of GPS sources, and this lack of evidence gives further support to the youth scenario (e.g., Morganti 2008, p. 210; see however, García-Burillo et al. 2007). At the same time, recent broad-band lowfrequency radio observations reveal an inhomogeneous absorber surrounding compact radio lobes in a few CSOs (e.g., Callingham et al. 2015; Tingay et al. 2015), implying that complex interactions between expanding jets and the surrounding medium do take place. X-ray observations of CSOs can potentially resolve this issue by providing evidence in favor of or against the presence of dense obscuring matter able to frustrate the jets in the youngest sources.

In general the GPS sources are faint in X-rays, and studying them in this waveband became possible only during the last decade, thanks to the Chandra X-ray Observatory (e.g., Siemiginowska et al. 2008; Kunert-Bajraszewska et al. 2014) and XMM-Newton (e.g., Guainazzi et al. 2006; Vink et al. 2006; Tengstrand et al. 2009). The early X-ray results indicate that the GPS X-ray emission could be intrinsically absorbed, although the absorption properties of the GPS sources are similar to those found in general source populations. The intrinsic GPS X-ray radiation could be related to the accretion, to expanding radio lobes, to relativistic jets, or in some cases to the thermally hot medium found in the central regions of the host galaxy (Guainazzi et al. 2006; Stawarz et al. 2008; Ostorero et al. 2010; Migliori et al. 2011, 2012, 2014).

However, only a few CSO sources with known redshift and measured age have been observed in X-rays (e.g., Risaliti et al. 2003; Guainazzi et al. 2006; Vink et al. 2006). Thus, we initiated ourChandra program to study the CSO sample defined by requiring the availability of kinematic age measurements and $z<1$ (Section 2). Our main goal was to establish the X-ray properties of these CSOs, determine the origin of their X-ray emission, and study the properties of the environment into which they are expanding. We describe our methods of X-ray data analysis in Section 3, and present the results of X-ray analysis in Section 4. We discuss the implications of the CSO X-ray properties for the models of the high-energy processes in compact radio sources in Section 5 and conclude our findings in Section 6. We use the most recent constraints on the cosmological parameters to convert the observed fluxes into luminosities (Hinshaw et al. 2013; $H_{0}=69.3 \mathrm{~km} \mathrm{~s}^{-1} \mathrm{Mpc}^{-1}, \quad \Omega_{m}=0.287 \quad$ implemented as WMAP9 in the astropy.cosmology package, Astropy Collaboration et al. 2013).

\section{SAMPLE}

We compiled from the literature a sample of 16 CSO sources with redshift and expansion velocity of the radio hot spots available at the time of our proposed Chandra project. Table 1 lists these sources and their general radio properties (the linear size, $\mathrm{LS} \simeq 2-120 \mathrm{kpc}$; the expansion velocity, $v \simeq 0.07 c-0.4 c$; and the age inferred through the kinematic argument, $\tau \sim$ $100-3000$ yr).

We obtained new Chandra observations of 10 CSOs. We observed the six CSOs with no prior X-ray information (5 ks exposures). Additionally, we imaged with Chandra the two sources observed previously with BeppoSAX $(1946+708,5 \mathrm{ks}$; $1934-63,20 \mathrm{ks})$ or with XMM-Newton $(0710+439,38 \mathrm{ks}$;
$1607+26,38 \mathrm{ks})$. The goal of the long (20-40 ks) exposures was to study in detail the CSO environments through the highresolution spatial and spectral analysis allowed by the Chandra data. Table 2 contains the log of our Chandra observations. It includes also $1511+0518$ with archival $2 \mathrm{ks}$ Chandra data.

Figure 1 shows the distribution of the kinematic ages of CSOs for 16 sources in our pilot study and seven additional sources from An \& Baan (2012) with their ages measured after our Chandra program was completed. The uncertainties on the age measurements are typically within $20 \%-40 \%$ and related to the quality of the radio monitoring data. Note that our X-ray sample contains all the youngest CSOs with ages $<400 \mathrm{yr}$.

In summary, Table 1 contains a complete list of CSO sources with derived redshift and kinematic age that have been observed in X-rays to date, while Table 2 contains the sources analyzed in this paper. With our Chandra program, we have increased the size of the CSO X-ray sample by $\sim 68 \%$.

\section{CHANDRA X-RAY OBSERVATIONS AND DATA ANALYSIS}

The ChandraACIS-S data were collected during the 2010-2011 epoch (see Table 2 for details). All targets were placed at the aim point on the back-illuminated ACIS CCD (S3). The observations were made in VFAINT mode with $1 / 8$ CCD readout to avoid pileup if sources were to be bright. All 10 targets were detected by Chandra with numbers of counts between 3 and 1677.

The X-ray data analysis was performed with the CIAO version 4.6 software (Fruscione et al. 2006) using CALDB version 4.4. We processed the data by running the CIAO tool acis-process-events and applied the newest calibration files, filtered VFAINT background events, and ran a sub-pixel event-repositioning algorithm (and set pix_adj=EDSER). This final step provides X-ray image data with the highest angular resolution for the most up-to-date ACIS-S calibration. All spectral modeling was done in Sherpa ${ }^{9}$ (Freeman et al. 2001; Refsdal et al. 2009). We used the Cash and Cstat fitting statistics (Cash 1979) and the Nelder-Mead optimization method (Nelder \& Mead 1965).

\subsection{Image Analysis}

We inspected the Chandra data using ds 9 and visually confirmed the location of each source, and also defined the source and background regions for further spectral extractions. Figure 2 displays the $\sim 10^{\prime \prime} \times 10^{\prime \prime}$ ACIS-S images of all the targets (denoted in the panels with green circles), except for $0116+319$, which is detected with three counts only ( $p$-value $\ll 0.01){ }^{10}$ The target is the brightest source in the image. We stress that due to the different redshifts of our CSO sources, the Chandra observations probe physical scales that differ by $\sim 1.5$ orders of magnitude (0.4-11.2 kpc; Figure 2).

Hints of extended X-ray emission in the three sources with the highest numbers of counts $(0710+0439,1718-649,1934$ -638 ), or a secondary X-ray source (in $1607+268$ ), could be seen in the images. We investigated the significance of the extended X-ray emission using surface brightness analysis. We compared the observed surface brightness profiles to the point-

\footnotetext{
9 http://cxc.harvard.edu/sherpa/

10 The $p$-value is the probability that the observed counts are due to the Poisson background fluctuations. $p$-value $<0.01$ indicates a detection. It is derived via simulations.
} 
Table 1

CSOs with Known Redshift and Kinematic Age Measurements

\begin{tabular}{|c|c|c|c|c|c|c|c|}
\hline \# & $\begin{array}{l}\text { Source } \\
\text { Name }\end{array}$ & $z$ & $\begin{array}{l}\text { Size } \\
(\mathrm{pc})\end{array}$ & $\begin{array}{l}\text { Velocity } \\
\quad(c)\end{array}$ & $\begin{array}{l}\mathrm{Age}^{\mathrm{a}} \\
(\mathrm{yr})\end{array}$ & $\begin{array}{l}\text { Refs. } \\
\text { (Age) }\end{array}$ & $\begin{array}{c}\text { Refs. }^{\mathrm{c}} \\
\text { (X-ray Observations) }\end{array}$ \\
\hline 1 & $1718-649^{\mathrm{d}}$ & 0.014 & 2.0 & 0.07 & 91 & (1) & This work/C \\
\hline 2 & $1843+356$ & 0.763 & 22.3 & 0.39 & 180 & (2) & This work/C \\
\hline 4 & $0035+227$ & 0.096 & 21.8 & 0.15 & 450 & (1) & This work/C \\
\hline 5 & $0116+319^{\mathrm{e}}$ & 0.059 & 70.1 & 0.45 & 501 & (1) & This work/C \\
\hline 6 & $0710+439$ & 0.518 & 87.7 & 0.30 & 932 & (2) & This work/C; $(6 / N)$ \\
\hline 9 & $1934-638$ & 0.183 & 85.1 & 0.17 & 1603 & (1) & This work/C; $(7 / \mathrm{B})$ \\
\hline 10 & $1607+26^{\mathrm{f}}$ & 0.473 & 240 & 0.34 & 2200 & (3) & This work/C; $(8 / \mathrm{N})$ \\
\hline 11 & $1511+0518$ & 0.084 & 7.3 & 0.15 & 300 & (4) & $(9 / \mathrm{C})$ \\
\hline 12 & $1245+676$ & 0.107 & 9.6 & 0.16 & 188 & (1) & $(10 / N)$ \\
\hline 13 & $\mathrm{OQ}+208^{\mathrm{g}}$ & 0.077 & 7.0 & 0.10 & 219 & (5) & $(11 / \mathrm{N})$ \\
\hline
\end{tabular}

Notes.

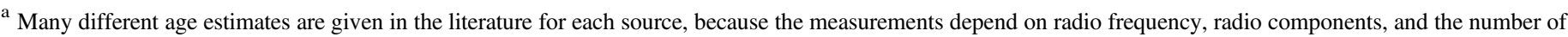

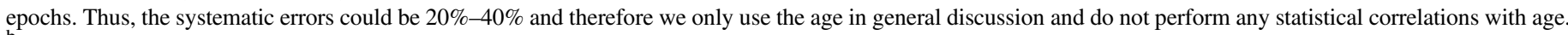
b (1) Giroletti \& Polatidis (2009), (2) Polatidis \& Conway (2003), (3) Nagai et al. (2006), (4) An et al. (2012), (5) Luo et al. (2007).

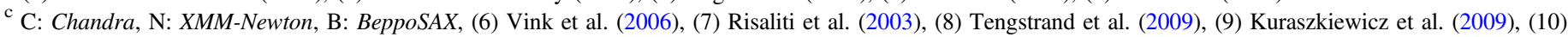
Watson et al. (2009), (11) Guainazzi et al. (2004).

d NGC 6328.

e $4 \mathrm{C}+31.04$.

f CTD 093.

g Mkn 668.

Table 2

Chandra Observations

\begin{tabular}{|c|c|c|c|c|c|c|c|c|}
\hline \# & $\begin{array}{l}\text { Source } \\
\text { Name }\end{array}$ & R.A. (J2000) & Decl. (J2000) & Date & Obsid. & $\begin{array}{l}\text { Exposure } \\
\text { (s) }\end{array}$ & $\begin{array}{c}\text { Total }^{\mathrm{a}} \\
\text { Counts }\end{array}$ & $\begin{array}{l}\text { Net }^{\mathrm{a}} \\
\text { Counts }\end{array}$ \\
\hline 1 & $1718-649$ & 172341.0 & -650036.6 & 2010 Nov 09 & 12849 & 4783 & 231 & $224.9 \pm 15.4$ \\
\hline 2 & $1843+356$ & 184535.1 & +354116.7 & 2010 Oct 26 & 12850 & 4783 & 11 & $10.8 \pm 3.3$ \\
\hline 4 & $0035+227$ & 003808.1 & +230328.4 & 2010 Oct 13 & 12847 & 4783 & 10 & $9.9 \pm 3.2$ \\
\hline 5 & $0116+319$ & 011935.0 & +321050.0 & 2010 Nov 05 & 12848 & 4742 & 3 & $\ldots$ \\
\hline 6 & $0710+439$ & $07 \quad 1338.2$ & +434917.2 & 2011 Jan 18 & 12845 & 37845 & 1679 & $1676.8 \pm 41.0$ \\
\hline 9 & $1934-63$ & 193925.0 & -634245.6 & $2010 \mathrm{Jul} 08$ & 11504 & 19793 & 362 & $361 \pm 19.0$ \\
\hline \multirow[t]{2}{*}{10} & $1607+26$ & 160913.3 & +264129.0 & 2010 Dec 04 & 12846 & 37845 & 213 & $212.5 \pm 14.5$ \\
\hline & secondary ${ }^{\mathrm{b}}$ & 160912.7 & +264117.5 & 2010 Dec 04 & 12846 & 37845 & 32 & $30.8 \pm 5.7$ \\
\hline 11 & $1511+0518^{\mathrm{c}}$ & 151141.2 & +05 1809.2 & 2003 May 18 & 4047 & 1994 & 49 & $48.8 \pm 7.1$ \\
\hline
\end{tabular}

Notes.

a Total and background-subtracted counts in a $r=1$.' 5 circle with energies between 0.5 and $7 \mathrm{keV}$.

b The secondary X-ray source resolved in our Chandra image.

${ }^{\mathrm{c}}$ Chandra archival data.

spread function (PSF) profile simulated by $\mathrm{CHART}^{11}$ that is expected for a point source.

\subsection{Spectral Analysis}

The X-ray spectra and corresponding calibration files (arf and $r m f$ ) were extracted with the CIAO script SPECEXTRACT for all the sources. We assumed a circular region of radius 1 !"5

\footnotetext{
${ }^{11}$ http://cxc.harvard.edu/chart/
}

centered on the position of each source for the spectral extraction. A local background was extracted assuming an annulus with inner and outer radii equal to 1.7 and $10^{\prime \prime}$, respectively. We compared the total number of counts and net counts for each source (Table 2). We assessed that the background was negligible in all cases $(<1$ count in the source extraction region for all targets, except for $0710+439$ with 2.1 counts, e.g., $<0.1 \%$ of the source counts), and therefore we ignored it during the spectral analysis (i.e., we modeled only the source spectra). 


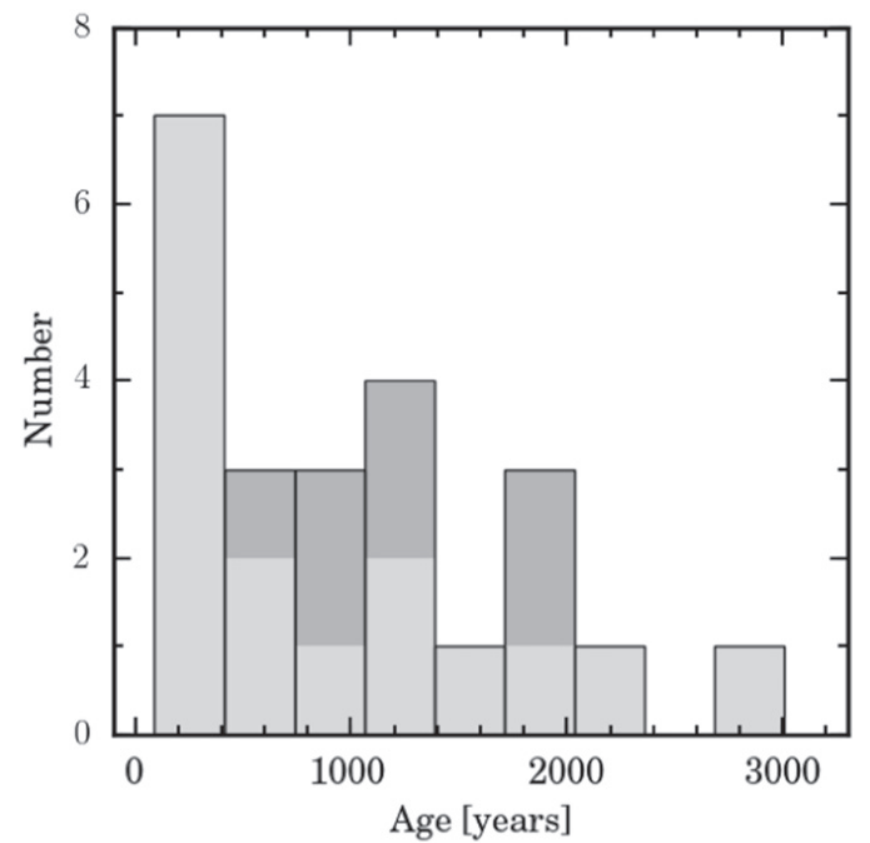

Figure 1. Distribution of the kinematic ages of CSOs for all available sources with age measurements to date. The distribution of sources with available $\mathrm{X}$-ray data (Table 1) is marked in light grey. The dark grey indicates additional CSOs from An \& Baan (2012) (except for PKS B1413+135 classified as coredominated) without X-ray data, with the ages derived after our Chandra program was completed. data:

We applied an absorbed power-law model to the spectral

$$
\begin{aligned}
N(E)= & A E^{-\Gamma} \exp \left\{-N_{\mathrm{H}}^{\mathrm{gal}} \sigma(E)-N_{\mathrm{H}}^{z} \sigma[E(1+z)]\right\}, \\
& \text { photons } \mathrm{cm}^{-2} \mathrm{~s}^{-1} \mathrm{keV}^{-1}
\end{aligned}
$$

where $A$ is the normalization at $1 \mathrm{keV}, \Gamma$ is the photon index of the power law, and $N_{\mathrm{H}}^{\mathrm{gal}}$ and $N_{\mathrm{H}}^{z}$ are the equivalent column densities for the two absorption componentsGalactic and intrinsic to the source. In the Sherpa nomenclature, this baseline spectral model was defined as xsphabs*xszphabs*powlawld and fit to the data in the energy range $0.5-7 \mathrm{keV}$. The Galactic absorption was always fixed at the respective value $N_{\mathrm{H}}^{\mathrm{gal}}$ listed for each source in Table 3, while the intrinsic absorption column was allowed to vary. The photon index $\Gamma$ was fixed at 1.7 (a typical value for an unabsorbed AGN with $N_{\mathrm{H}}<10^{22} \mathrm{~cm}^{-2}$, Burlon et al. 2011) while modeling the sources with a low number of counts, $<12$, in order to obtain a limiting value or detection of the intrinsic absorption. The range of the photon index $1.4<\Gamma<2.0$ results in the intrinsic column density being consistent to within $1 \sigma$ with the values listed in Table 3 . The photon index was a free parameter in sources with a larger number of counts.

\section{RESULTS}

We detected X-ray emission associated with all the CSOs in our Chandra sample. However, because the majority of our Chandra observations were short, designed for X-ray detections, detailed analysis was possible for only about half of the sources in the sample. In the short $(\sim 5 \mathrm{ks})$ observations the detected source counts ranged from 3 to 231 counts (see Table 2). The background in such a short Chandra observation of a point source is very low, and even three counts in $0116+319$ constitute a detection at a high significance level (p-value $<0.01$ ). However, we can only provide a limiting flux in this case (see Table 3), while for the other sources we were able to extract the Chandra spectrum and apply parametric models.

The three longer exposures provided data of high enough sensitivity to explore the CSOs' X-ray environment on arcsecond scales. We extracted the X-ray surface brightness profiles assuming that the annular regions centered on the position of the radio source quantified the presence of X-ray emission outside the central point source. In two cases, 0710 +0439 and 1934-638, the profiles are consistent with the background level outside a radius of $\sim 6^{\prime \prime}$. The residuals in the innermost circular regions deviate slightly from zero, suggesting the possibility of an additional emission component. However, the formal significance test gives a $p$-value of 0.33 , which does not allow us to reject the null hypothesis that the source is point-like. We concluded that in these two sources the observed X-ray emission originates in an unresolved region within $r<1$ !. 5 (corresponding to $<9.5 \mathrm{kpc}$ and $<4.7 \mathrm{kpc}$ for each source, respectively). There is no extended X-ray emission on large scales detected above the background level of 0.0048 counts $\operatorname{arcsec}^{-2}$.

The surface brightness profile for the third object, 1718 -649 , is shown in Figure 3. An excess of the observed emission above the PSF profile is visible at distances within $2^{\prime \prime}-6^{\prime \prime}(0.6-1.8 \mathrm{kpc})$ of the radio source position, indicating extended X-ray emission present in this source. We add another model component (the standard beta model, betald in Sherpa; King 1962) to account for this emission. We obtained a $1 \sigma$ lower limit of $\beta>0.55$ in our best-fit model. Deep Chandra observations are needed to better constrain the properties of this emission.

In general, our baseline absorbed power-law model provided a good description of the X-ray continua of the CSO sources. The modeling results are given in Table 3 , which displays the Galactic and intrinsic absorption columns, photon index, and the unabsorbed flux in the soft $(0.5-2 \mathrm{keV})$ and hard (2-10 keV; extrapolated from the model fitted over the $0.5-7 \mathrm{keV}$ band) $\mathrm{X}$-ray bands. The uncertainties are given as $1 \sigma$ for one interesting parameter. In seven CSOs the photon index was a free parameter and we obtained $\Gamma \simeq 1.4-1.7$, with the exception of $2021+614$ and $1511+0518$ where $\Gamma \simeq 1$. The intrinsic absorption component was required at $>2 \sigma$ confidence level in three CSOs $(0710+439,1946+708$, and $0035+227$; note though that in $0035+227$ the photon index was fixed at 1.7) with $N_{\mathrm{H}}^{z} \simeq(0.6-1.7) \times 10^{22} \mathrm{~cm}^{-2}$. In three other cases we were able to derive only upper limits on the intrinsic column densities (but see Section 4.2). The X-ray spectra of the remaining five sources are suggestive of the presence of an intrinsic absorbing neutral hydrogen column with $N_{\mathrm{H}}^{z} \simeq 10^{21}$ $10^{22} \mathrm{~cm}^{-2}$, although at low statistical significance.

Even though we detected an extended X-ray emission in 1718-649, the CSO with the lowest redshift in our sample, we find that the X-ray spectrum of this source is quite well modeled with an absorbed power law. However, our Chandra spectrum has only $224 \pm 15$ counts (see Table 3 ) and any detailed analysis of potential contributions from emission associated with the extended medium would require X-ray data of higher quality. 

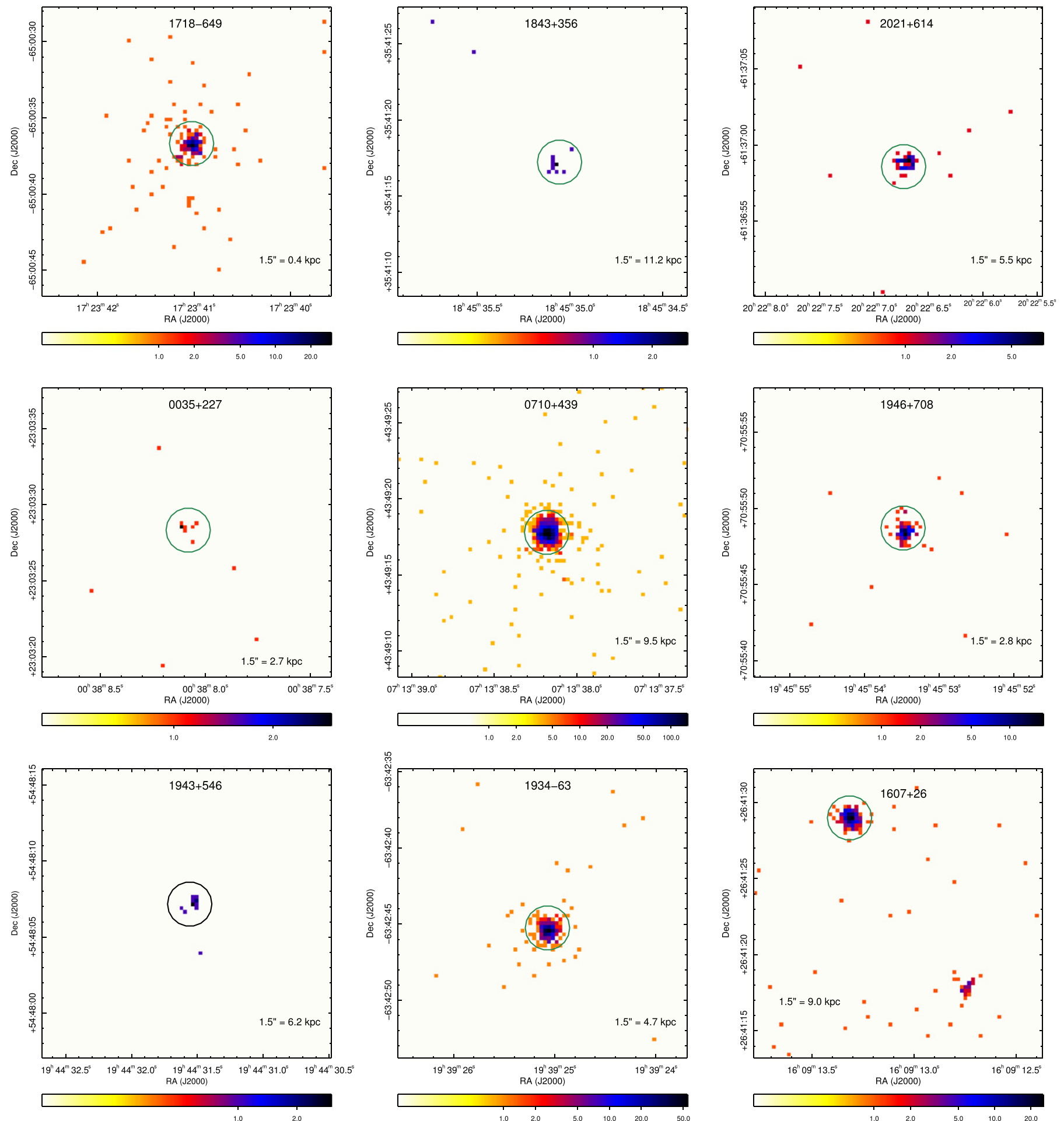

Figure 2. ACIS-S images of CSOs sources observed in our Chandra program (Table 2), except for 0116+319 detected with only three counts. X-ray events in the energy range between 0.5 and $7 \mathrm{keV}$ are displayed. The pixel size is set to 0 !! 264 and each panel shows a region of 9 !' $84 \times 9$ !" 84 . A circular region of radius 1 !! 5 is plotted around each source and its corresponding scale in kiloparsecs is marked in each panel.

Below we provide a detailed description of the X-ray results for three particular CSOs in our sample: $0710+439$ has a hardband narrow emission line (Section 4.1); 2021+614 and 1511 +0518 have an extremely hard X-ray photon index compared to typical AGNs (Section 4.2); and $1607+26$ is accompanied by a secondary X-ray source resolved within the prior XMMNewton extraction region (Section 4.3). We also comment on
1934-63 and 1946+708 observed previously with BeppoSAX and revisited with our Chandra program (Section 4.4).

\section{1. $0710+439$}

The photon index and the amount of intrinsic absorption in our Chandra spectrum of $0710+439$ agree very well with the earlier XMM-Newton results of Vink et al. (2006). The source 
Table 3

Chandra Best Fit Model Parameters

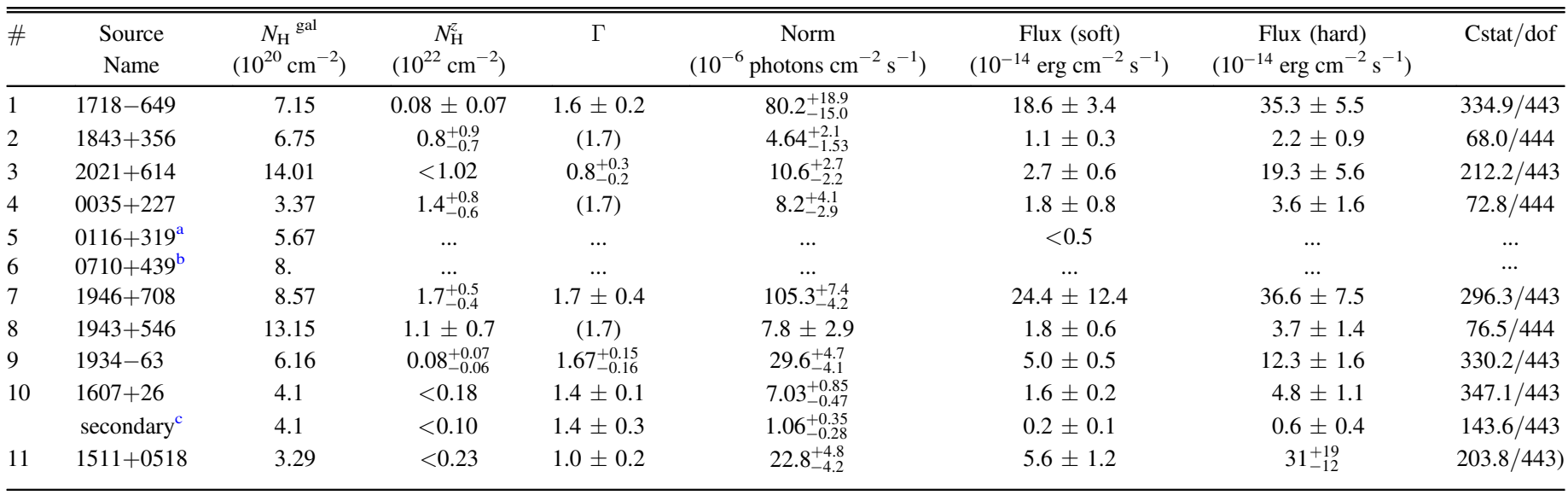

Notes. Unabsorbed flux in the observed energy range (soft: $0.5-2 \mathrm{keV}$; hard: $2-10 \mathrm{keV}$, extrapolated from the model fitted over the range $0.5-7 \mathrm{keV}$ ). All uncertainties are given as $1 \sigma$ for one interesting parameter. Upper limits are $3 \sigma$ limits;

${ }^{a}$ Three counts were detected in the source region and no model fitting was performed for this source. We list the $3 \sigma$ flux limit estimated using the s $r \mathrm{cflux}$ tool, which performs simulations (Kashyap et al. 2010).

${ }^{\mathrm{b}}$ See Table 4 .

${ }^{\mathrm{c}}$ The secondary X-ray source resolved in our Chandra image with $N_{\mathrm{H}}$ limit at $z=0$.
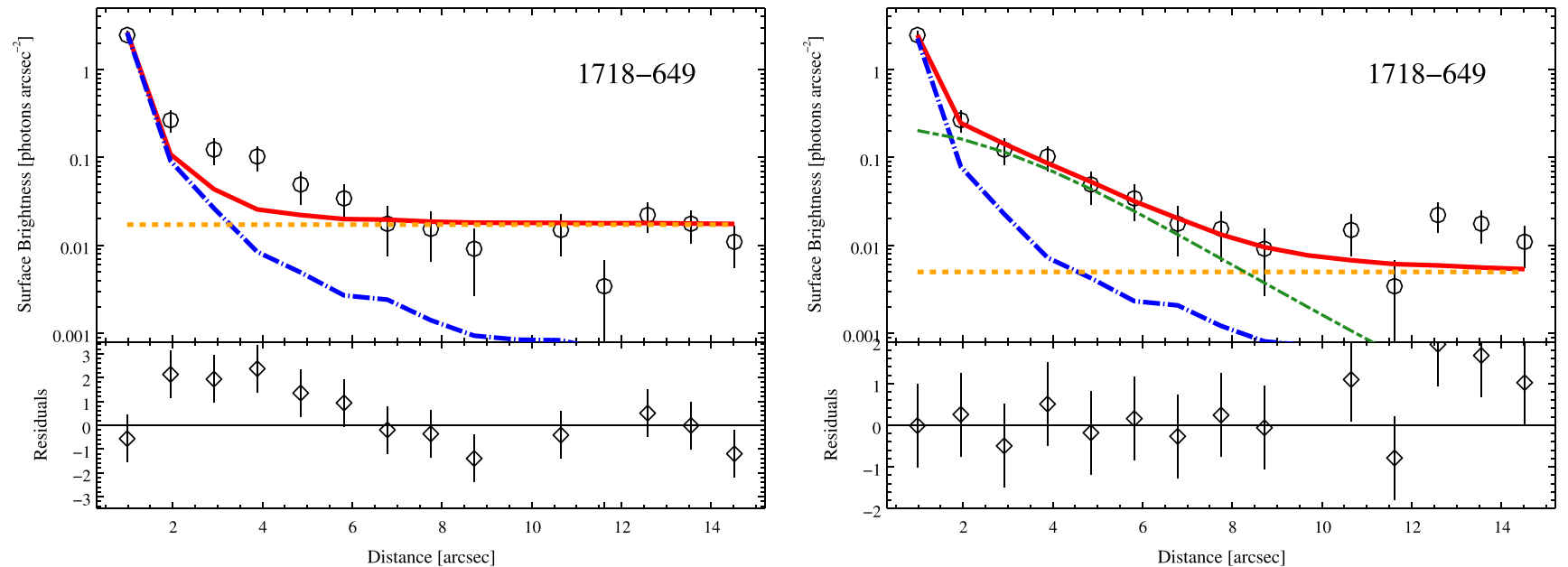

Figure 3. X-ray surface brightness profile of 1718-649. Different model components include the Chandra PSF profile (blue long dashed curves), constant background (orange dotted lines), and the additional standard beta model with $\beta>0.55$ (green short dashed curve); thick solid red curves denotes the total model fitted to the data (without and with the beta component in the left and right panels, respectively), and the corresponding residuals are given in the lower panels.

did not vary between the XMM-Newton and Chandra observations, showing consistent $2-10 \mathrm{keV}$ flux of about $4.1 \times 10^{-13} \mathrm{erg} \mathrm{cm}^{-2} \mathrm{~s}^{-1}$. However, we find clear residuals in the hard X-ray band suggestive of the presence of an emission line (Figure 4, top left). To account for this feature we added a Gaussian component to the absorbed power-law spectral model (Model B in Table 4; Figure 4, top right). The line is unresolved ( $\sigma=10 \mathrm{eV}$; fixed) with the best-fit line energy of $E=6.62 \pm 0.04 \mathrm{keV}$ (rest frame) and the equivalent width $\mathrm{EW}=154_{-58}^{+65} \mathrm{eV}$. Visual inspection of the $X M M$ Newton modeling presented by Vink et al. (2006) tentatively hints at the presence of an emission line at similar energy also in the XMM-Newton data set.

Emission lines at the energies within $\sim 6.4-6.9 \mathrm{keV}$ due to iron are common in X-ray spectra of accreting black holes (Krolik \& Kallman 1987; George \& Fabian 1991; Zycki \& Czerny 1994), including radio-quiet and non-blazar radio-loud
AGNs (e.g., Hardcastle et al. 2009, Winter et al. 2009; Fukazawa et al. 2011; Zhou et al. 2011) and Galactic black hole binaries (e.g., Done et al. 2007). In particular, a narrow fluorescent neutral $\mathrm{Fe} \mathrm{K} \alpha$ line at $6.4 \mathrm{keV}$ (rest frame) seems to be omnipresent in the spectra of radiatively efficient AGNs (Bianchi et al. 2004; Nandra et al. 2007; Iwasawa et al. 2012, and references therein), and is believed to originate from cold matter, e.g., a molecular torus, illuminated by hard X-rays.

Narrow $\sim 6.6-6.9 \mathrm{keV}$ emission lines from ionized iron have been observed, e.g., in luminous Palomar Green quasars, including the radio-loud source Mrk 1383 (Porquet et al. 2004), and in a substantial fraction of Seyfert 1 galaxies (Patrick et al. 2012). These lines could be emitted by a hot ionized region of an accretion disk illuminated by an external X-ray source (e.g., Matt et al. 1993; Różańska et al. 2002), by a distant gas photoionized by the nuclear illumination (e.g., 

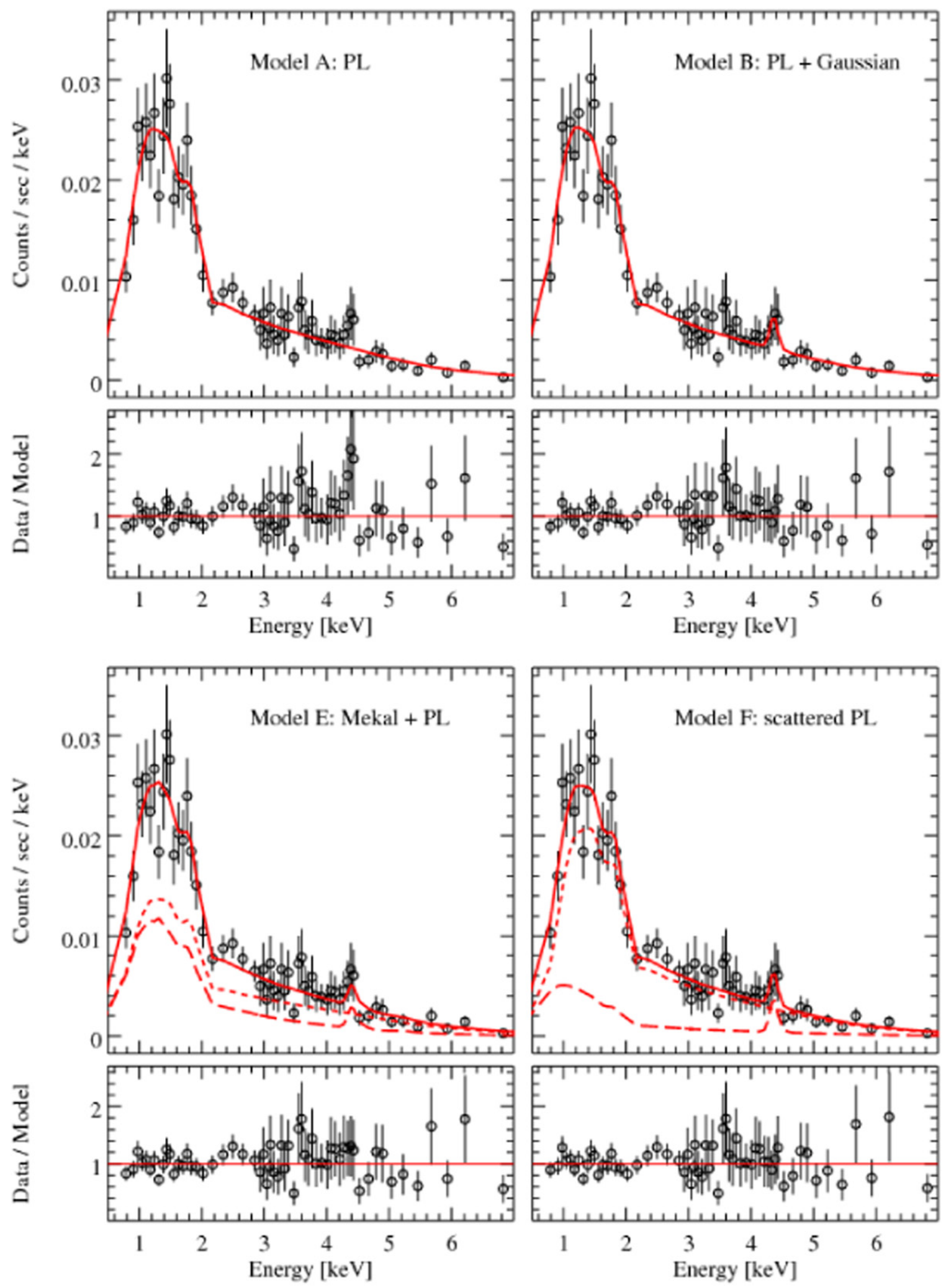

Figure 4. Spectral modeling of 0710+0439. Each panel shows the Chandra data (open circles), total models (solid; see Table 4), model components, and the ratio of the data to the best fitting model. Top: an absorbed power law without the emission line (left, Model A) and with the emission line (right, Model B). Bottom: twocomponent models consisting of (left, Model E) thermal emission of diffuse plasma (long dashed) and a direct power law (short dashed), and (right, Model F) a direct power law (short dashed) and a scattered power law plus a Gaussian emission line (long dashed). The data have been grouped for presentation purposes by requiring signal-to-noise ratios of 5 and 3 , below and above $3 \mathrm{keV}$, respectively. 
Table 4

Spectral Models for $0710+439$

\begin{tabular}{|c|c|c|c|c|c|c|c|c|c|c|}
\hline \# & $\begin{array}{c}\text { Model } \\
\text { Description }^{\mathrm{a}}\end{array}$ & $\begin{array}{c}N_{\mathrm{H}}(z) \\
\left(10^{22} \mathrm{~cm}^{-2}\right)\end{array}$ & $\Gamma$ & $\begin{array}{c}k T \\
(\mathrm{keV})\end{array}$ & $f_{\mathrm{sc}}$ & $\begin{array}{c}E_{\mathrm{Fe} \mathrm{xxv}} \\
(\mathrm{keV})\end{array}$ & $\begin{array}{l}\text { EW } \\
(\mathrm{eV})\end{array}$ & $\begin{array}{l}\text { Flux } \\
\text { (soft) }\end{array}$ & $\begin{array}{l}\text { Flux } \\
\text { (hard) }\end{array}$ & Cstat/dof \\
\hline A & PL & $0.58 \pm 0.08$ & $1.59 \pm 0.07$ & $\ldots$ & $\ldots$ & $\ldots$ & $\ldots$ & $19.7_{-1.5}^{+1.5}$ & $42.2_{-5.5}^{+6.3}$ & $490.3 / 443$ \\
\hline B & PL and $\mathrm{G}$ & $0.61_{-0.08}^{+0.04}$ & $1.64_{-0.07}^{+0.03}$ & $\ldots$ & $\ldots$ & $6.62 \pm 0.04$ & $154_{-58}^{+65}$ & $20.2_{-1.6}^{+1.6}$ & $41.0_{-5.5}^{+6.3}$ & $480.3 / 441$ \\
\hline $\mathrm{C}$ & TB and $\mathrm{G}$ & $0.49_{-0.06}^{+0.07}$ & $\ldots$ & $13.6_{-2.8}^{+2.6}$ & $\ldots$ & $6.63 \pm 0.04$ & $164_{-58}^{+64}$ & $18.0_{-0.7}^{+0.7}$ & $37.0_{-3.2}^{+2.2}$ & $475.8 / 441$ \\
\hline D & PL and Apec & $0.58 \pm 0.08$ & $1.42_{-0.20}^{+0.14}$ & $5.4 \pm 1.5$ & ... & ... & ... & $19.7_{-2.8}^{+2.9}$ & $40.1_{-5.3}^{+5.6}$ & $485.0 / 441$ \\
\hline $\mathrm{E}$ & PL and Mekal & $0.56 \pm 0.08$ & $1.39_{-0.23}^{+0.17}$ & $5.2_{-1.3}^{+2.2}$ & $\ldots$ & $\ldots$ & $\ldots$ & $19.7_{-2.9}^{+2.9}$ & $40.4_{-5.5}^{+5.7}$ & $484.4 / 441$ \\
\hline
\end{tabular}

Notes.

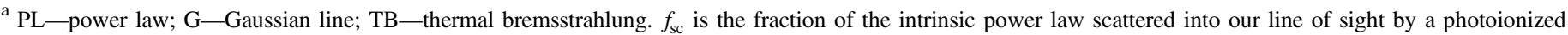

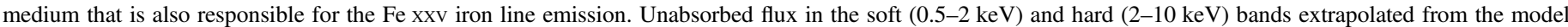

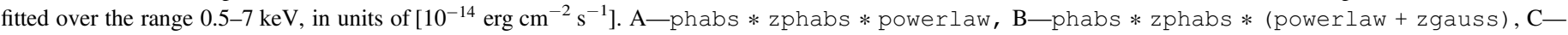

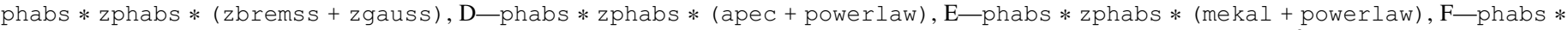

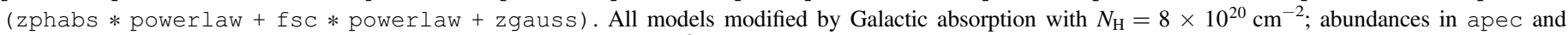
mekal models fixed at 0.5 solar value; mekal density fixed at $1 \mathrm{~cm}^{-3}$; width of the Fe line fixed at $\sigma=0.01 \mathrm{keV}$.

${ }^{\mathrm{b}} \mathrm{EW}$ calculated with respect to the unabsorbed scattered power law, fsc * powerlaw.

Bianchi \& Matt 2002), or by a diffuse thermal plasma at $k T \gtrsim 5 \mathrm{keV}$ (Smith et al. 2001).

The best-fit parameters of the line detected in $0710+439$ assuming Model B (see Table 4) are consistent with the first scenario and with the He-like Fe Xxv line emission ${ }^{12}$ in a system with an inclination angle $\cos i \simeq 0.5-0.6$ and an accretion rate $\dot{M} \simeq 0.4 \dot{M}_{\text {Edd }}$ (Matt et al. 1993). However, in order to study the origin of this emission line in more detail we also tested four other spectral models of increased complexity that are typically considered for AGN X-ray emission (Table 4). Given the quality of the current data all the models are statistically equivalent, but considering the range of the best-fit parameters we can potentially rule out some of them.

First, we parameterized the X-ray continuum with a thermal bremsstrahlung model (zbremss) and kept the Gaussian profile to fit the emission feature (Model $\mathrm{C}$ in Table 4). We found a statistically good fit, but with a relatively high temperature, $k T \sim 14 \mathrm{keV}$. Next, we replaced the bremsstrahlung continuum with a single-temperature plasma (mekal) model (metal abundances fixed at half the solar value) and found that at this high temperature the strength of the ionized Fe lines was overpredicted, especially at the energy of the fully ionized Fe XXVI, while the data did not provide evidence for the presence of the Fe XXVI line. A similar result was obtained when we used the apec plasma model instead of the mekal model. Hence we concluded that single-component thermal models are not likely given the physical properties required by the best-fit parameters. Therefore, we tried a two-component model, consisting of a power law and either mekal or apec (Models D and E in Table 4). We obtained the best-fit plasma temperature $k T \sim 5 \mathrm{keV}$, and a hard photon index, $\Gamma=1.4$ 1.5 , which are consistent with the prediction of jet/lobe emission.

Finally, we tested a model in which the spectrum is composed of the intrinsically absorbed primary emission (intrinsic emission modeled with a power law) and an unabsorbed fraction of the same emission scattered by a warm

\footnotetext{
12 The line is most probably a blend of unresolved resonance $(6.700 \mathrm{keV})$, intercombination $(6.682 \mathrm{keV}$ and $6.668 \mathrm{keV})$, and forbidden $(6.637 \mathrm{keV})$ lines.
}

medium. The warm medium is also producing the iron emission line (Model $\mathrm{F}$ in Table 4). In this scenario the photon index is $\Gamma=1.75_{-0.10}^{+0.11}$, the primary continuum is absorbed with a column of $N_{\mathrm{H}}^{z} \simeq 10^{22} \mathrm{~cm}^{-2}$, and the scattering fraction is $\sim 13 \%$. The EW of the line (computed with respect to the scattered continuum) is $\sim 1.4 \mathrm{keV}$, in agreement with the values found by Bianchi \& Matt (2002) for column densities of the order of a few $\times 10^{21} \mathrm{~cm}^{-2}$ (see their Figure 4). The best-fit parameters of the models A, B, E, and F, and the ratio of the data to the models, are presented in Figure 4. We conclude that with the present data we are not able to state whether the soft $\mathrm{X}$-rays and the He-like iron line in $0710+439$ are due to scattered nuclear emission (Models B or F) or thermal emission of the ISM heated through interactions with the expanding jet (Models D and E).

The neutral iron $\mathrm{K} \alpha$ line has been previously reported in CSO sources observed with XMM-Newton (OQ 208, Guainazzi et al. 2004; and 1607+26, Tengstrand et al. 2009), while Risaliti et al. (2003) detected neutral and highly ionized $(E \simeq 6.9 \mathrm{keV})$ iron emission lines in 1934-638 and 1946+708 (respectively) using BeppoSAX. These two emission lines are not present in the low-count Chandra spectra ${ }^{13}$ of $1607+26$ (see Section 4.3) and of the two BeppoSAX sources (see Section 4.4).

Müller (2015) detected a narrow emission line in a CSO candidate (PMN J1603-4904), and derived the redshift of the source assuming that the most likely rest-frame energy of the line is $6.4 \mathrm{keV}$. The presence of a mildly ionized Fe line in the spectrum of $0710+439$ indicates that there might be a systematic error in the redshift derivation of PMNJ1603 -4904 because of a range of ionization states of iron producing the emission lines in the CSO sources.

Lastly, we note that $0710+439$ has the highest number of counts (1679) among the sources in our sample. The remaining CSOs were detected with 3-362 counts. A higher number of

\footnotetext{
13 -values of 0.9 for $1934-63$ and 0.4 for $1946+708$ when testing for the line with the null hypothesis of the power-law model, and using the simulations as described in Protassov et al. (2002) (plot_pvalue in Sherpa).
} 
counts inChandra spectra is needed for detecting narrow spectral features.

\section{2. $2021+614$ and $1511+0518$}

The absorbed power-law model applied to two CSOs, 2021 +614 and $1511+0518$, resulted in an unusually hard photon index of $\Gamma=0.8 \pm 0.3$ and $\Gamma=1.0 \pm 0.2$ respectively. Typically, such a low value indicates a strong nuclear obscuration able to cover the source of the primary X-ray radiation completely. The observed spectrum is then dominated by a Compton reflection hump (and an accompanying neutral iron line) from distant material, presumably a molecular torus, resulting in an artificially low photon index. AGNs with these properties are called "Compton-thick," and the amount of hydrogen in the absorbing column required to distort the X-ray spectrum in this manner is $N_{\mathrm{H}} \gtrsim 10^{24} \mathrm{~cm}^{-2}$ (e.g., Comastri 2004, p. 245). The first radio-loud Compton-thick AGN was found in OQ 208, a CSO source observed with XMM-Newton by Guainazzi et al. (2004). Tengstrand et al. (2009) speculated that $1607+26$ is also a Compton-thick CSO. They based their argument on the photon index $\Gamma=0.4 \pm 0.3$ obtained when an absorbed power-law model was applied to the XMM-Newton data on this source. By analogy with OQ 208 and $1607+26$, it is likely that $2021+614$ and $1511+0518$ are Compton-thick CSO candidates. Our result on the intrinsic absorption in these sources would then be significantly underestimated because in our fit we do not account for the column density that would be needed to absorb the primary continuum. Indeed, when we fit the data of $2021+614$ with the sum of an intrinsically absorbed power law and an unabsorbed reflection (pexrav) component we obtain Cstat/dof $=209 / 442, N_{\mathrm{H}}^{z} \gtrsim 9.5 \times 10^{23} \mathrm{~cm}^{-2}$. The resulting photon index of the underlying power law would, however, be very soft, $\Gamma=3.3 \pm 0.3$, and the unabsorbed intrinsic $2-10 \mathrm{keV}$ luminosity would increase by an order of magnitude, making $2021+614$ the second CSO in our sample (in addition to $0710+439$ ) to exceed $L_{\mathrm{X}} \sim 10^{44} \mathrm{erg} \mathrm{s}^{-1}$ (comparable to the Eddington luminosity of a $10^{6} \mathrm{M}_{\odot}$ black hole).

In $1511+0518$ the Compton-thick model results in Cstat/dof $=198.2 / 443, N_{\mathrm{H}}^{z}=3.8_{-1.3}^{+4.0} \times 10^{23} \mathrm{~cm}^{-2}, \Gamma=3.8_{-0.4}^{+0.3}$, and $L_{\mathrm{X}} \sim 3 \times 10^{43} \mathrm{erg} \mathrm{s}^{-1}$.

On the other hand, we do not see any evidence for the presence of a neutral iron line in the spectra of $2021+614$ and $1511+0518$. The line is unconstrained in $1511+0518$. In 2021 +614 we can only derive an upper limit on the EW of this line, $\mathrm{EW}<0.7 \mathrm{keV}$, inconsistent with the expected range derived by Matt et al. (1996; $\mathrm{EW}=1.3-2.7 \mathrm{keV})$. Thus, it is interesting to note that the low X-ray photon index and the low intrinsic absorption can be understood in the context of a non-thermal X-ray lobe emission model for the GPS/CSO sources (Stawarz et al. 2008; Ostorero et al. 2010).

Unfortunately, the quality of our present Chandra data is not sufficient to discriminate between the Compton-thick absorption and X-ray lobe emission scenarios for X-ray emission in $2021+614$ and $1511+0518$ (only 54 and 49 counts collected in $5 \mathrm{ks}$ and $2 \mathrm{ks}$ Chandra observations, respectively). Deeper X-ray observations with Chandra or XMM-Newton accompanied by the hard $>10 \mathrm{keV}$ band exposures $(N u S T A R)$ would be needed in order to resolve this issue.

\section{3. $1607+26$}

Owing to the superior spatial resolution of Chandra we resolve the source region of $1607+26$ observed with XMMNewton by Tengstrand et al. (2009) into two point-like X-ray sources (see Figure 2). The location of $1607+26$ matches the position of the upper left source visible in Figure 2 with 213 counts detected. The secondary source separated by $\sim 13$." 5 (lower right corner in Figure 2) has 32 counts and its spectrum could be modeled with an absorbed power law (assuming only Galactic absorption since the redshift to the source is unknown) with a photon index $\Gamma=1.4 \pm 0.3$ (see Table 3 ). This means that the XMM-Newton spectrum obtained by Tengstrand et al. (2009) represents a combination of emission from $1607+26$ and from the secondary source. We detect an order-ofmagnitude decrease between the hard X-ray fluxes of $X M M$ Newton $\left(4.2 \times 10^{-13} \mathrm{erg} \mathrm{s}^{-1} \mathrm{~cm}^{-2}\right)$ and Chandra $\left(4.8 \times 10^{-14} \mathrm{erg} \mathrm{s}^{-1} \mathrm{~cm}^{-2}\right)$. This change is larger than the sum of the fluxes from the two sources, and it is larger than the absolute calibration uncertainties between the Chandra and XMM-Newton satellites (Tsujimoto et al. 2011). Therefore, we concluded that the X-ray flux of one or both of the sources decreased during the interval of aobut three years between the two observations.

We searched the SDSS, 2MASS, and FIRST catalogs to check the identification of the secondary X-ray source, but did not find any corresponding emission. If the source were associated with $1607+26$ then it would be located $\sim 80 \mathrm{kpc}$ away along the axis of the CSO radio structure, on the extension of its radio jet (see Nagai et al. 2006 for a discussion of the CSO radio morphology). It is interesting to speculate that the observed secondary X-ray source might be a hot spot due to the past radio activity in the framework of the intermittent scenario for the GPS sources. However, assuming the expansion velocity of $0.34 c$ (Table 1 ) the age of this feature would be $\sim 1$ Myr, while the terminal hot spots are expected to die very quickly, on timescales $\sim 0.1 \mathrm{Myr}$ once the jet switches off (Carilli et al. 1988). Therefore, the nature and the association of this secondary source with $1607+26$ remain unclear.

Contrary to Tengstrand et al. (2009), who reported $\Gamma=0.4 \pm 0.3$ in $1607+26$, we find $\Gamma=1.4 \pm 0.1$, an X-ray photon index that is fairly common in a population of nearby radio-quiet and radio-loud AGNs (e.g., Winter et al. 2009; Fukazawa et al. 2011). We do not detect any evidence of the neutral iron $\mathrm{K} \alpha$ line in the spectrum. This line, however, was required by the XMM-Newton data of Tengstrand et al. (2009) as part of their Compton reflection-dominated model for this source.

One explanation of this result could be that $1607+26$ is a transitional Compton-thick/thin AGN. Transitions between the Compton-thick and -thin regimes have previously been reported in the so-called "changing look" AGNs (see, e.g., Matt et al. 2003; Risaliti et al. 2005, 2010, 2011 and references therein). The Compton-thick/thin switches are thought to be due to an X-ray absorber made of clouds with $N_{\mathrm{H}} \simeq 10^{23}$ $10^{24} \mathrm{~cm}^{-2}$ crossing our line of sight on timescales between hours and weeks.

If the flux from the secondary source were constant between the two exposures, then $1607+26$ would have been $\gtrsim 10$ times brighter in the reflection-dominated state recorded with $X M M$ Newton than in our observation. This clearly contradicts the "changing look" AGN scenario. To reconcile the variability of 
the hard X-ray flux with the "changing look" hypothesis, 1607 +26 could not have contributed more than $\sim 200$ counts to the $X M M$-Newton data, meaning that the secondary X-ray source would have to be $\gtrsim 10$ times brighter than $1607+26$ in the XMM-Newton observation. This would imply that the X-rays detected and modeled by Tengstrand et al. (2009; including the iron line) originated from the secondary source rather than from $1607+26$, eliminating any spectral information about the state of $1607+26$ during the XMM-Newton observation. It would also indicate that the photon index of the secondary source changed from $\Gamma \sim 0.8$ to $\sim 1.4$, its iron line disappeared, and the hard flux increased by a factor of $\gtrsim 70$ between the two exposures. These spectral properties would be very difficult to explain, especially with no radio, optical, or $\gamma$-ray identification available at present for the secondary source. Thus, we reject the possibility that X-ray variability observed in $1607+26$ is due to the Compton-thick/thin switches.

The alternative explanations include fading of the nuclear and reflected $1607+26$ emission, or a heavy obscuration with a column density $\gtrsim 10^{24} \mathrm{~cm}^{-2}$, as a result of which Chandra would have observed thermal emission of the ISM. Finally, in the non-thermal scenario of Stawarz et al. (2008) and Ostorero et al. (2010) any potential intrinsic variability of the X-ray continuum is expected to take place on timescales much too long to account for the results of the Chandra observations of $1607+26$.

Clearly, future X-ray monitoring of this young radio source is needed in order to determine its nature and the properties of its X-ray variability.

\section{4. $1934-63$ and $1946+708$}

Risaliti et al. (2003) reported that 1934-63 and 1946+708 showed BeppoSAX spectra typical of the Compton-thick sources with $N_{\mathrm{H}}>2.5 \times 10^{24} \mathrm{~cm}^{-2}$, a strong $\mathrm{Fe} \mathrm{K} \alpha$ line, and the reflection hump. They also considered Compton-thin absorbers as a secondary explanation of the data. We have revisited both sources with Chandra. We could only obtain a $3 \sigma$ upper limit on the line equivalent width of $<0.96 \mathrm{keV}$ in $1934-63$ and of $<7.2 \mathrm{keV}$ in $1946+708$, assuming that the lines are unresolved $(\sigma=10 \mathrm{eV})$ and located at the rest energies, $6.3 \mathrm{keV}$ and $6.9 \mathrm{keV}$, respectively (as in Risaliti et al. 2003). Future, more sensitive X-ray observations are needed to constrain the parameters of these lines.

We measure a relatively low intrinsic absorption column density in both sources $\left(N_{\mathrm{H}}<2 \times 10^{22} \mathrm{~cm}^{-2}\right)$ and do not confirm their Compton-thick nature (see Table 3 ). We conclude either that the BeppoSAX spectra have been contaminated by other X-ray sources in the field, given the source extraction regions of 2 arcmin in the BeppoSAX observations, or that the Compton-thin model is a more appropriate explanation of the data, or that these sources belong to the AGN class that switches between the Compton-thick and -thin states.

\section{DISCUSSION}

We have investigated properties of the X-ray emission in a sample of 16 young radio sources with known redshift and kinematic age measurements of their radio structures. All sources in our sample have symmetric radio morphology, have been classified as CSOs, and have been associated with radio galaxies. They do not display strong AGN emission in the optical band, either because they do not contain an optical-UV bump characteristic of the AGN or because the optical emission has been absorbed by a dense medium in the central region of the host galaxy. We detected six of the sources in our sample for the first time in X-rays.

\subsection{Models for CSO X-Ray Emission}

The angular sizes of the CSO radio emission are of the order of milliarcseconds (e.g., Readhead et al. 1996b), and as such they are too small to be resolved with Chandra. As a result, $\mathrm{X}$-ray emission associated with any compact radio jets, lobes, or bow shocks potentially present around the lobes is integrated within the 1 .' 5 radius of the circular source extraction region, corresponding to $0.4-11 \mathrm{kpc}$ in our sources, depending on redshift. Consequently, the CSOs' $\mathrm{X}$-ray emission contains information on both the circumnuclear region (on sub-parsec and parsec scales) and the surrounding ISM (kiloparsec scale). Different physical processes may, thus, contribute to the observed X-ray emission (see, e.g., Siemiginowska 2009, for a review). The CSOs' $\mathrm{X}$-ray emission could arise from the AGN (X-ray corona), indicating the nature of the nucleus and its accretion state (Guainazzi et al. 2004; Vink et al. 2006; Siemiginowska et al. 2008; Tengstrand et al. 2009). However, if the absorption column is larger than $\sim 10^{24} \mathrm{~cm}^{-2}$, the nucleus could be hidden and the observed X-rays would be dominated by the thermal emission of the hot ISM from shocks driven by the expanding jet (Heinz et al. 1998; O’Dea et al. 2000) or by a reprocessed nuclear emission due to the reflection and/or scattering effects (e.g., Guainazzi et al. 2006). On the other hand, X-rays can also be emitted by compact radio lobes through inverse-Comptonization of local radiation fields (Stawarz et al. 2008; Ostorero et al. 2010; Migliori et al. 2012). These models result in different predictions of the X-ray photon index, the amount of intrinsic X-ray absorption, and the luminosity and could be tested with deep observations.

The CSOs in our sample are rapidly expanding, as demonstrated by their measured hot-spot expansion velocities spanning the range $0.07 c-0.4 c$ (Table 1, and references therein). Based on these velocities, the CSOs' kinematic age estimates range between $\sim 100$ and $3000 \mathrm{yr}$. These are, thus, the youngest radio sources that can provide information about the accretion state of supermassive black holes shortly after the onset of the jet formation, as well as about the properties of the environment into which the newly born jets are evolving, and with which they are interacting.

\subsection{Environment of $\mathrm{CSO}$}

Jets and lobes in young radio sources, confined to the central parts $(\sim 1 \mathrm{kpc})$ of their host galaxies, should interact with a denser and less homogeneous medium than their evolved analogs (tens to hundreds of kpc long). Indeed, our results support this idea. We detected multi-phase galactic environments consisting of diverse gaseous components co-spatial with the young expanding CSO radio structures: (1) the extended thermal component in 1718-649 (Section 3.1, Figure 3); (2) possibly an ionized diffuse plasma giving rise to the He-like Fe emission line in $0710+439$ (Section 4.1, Figure 4); (3) neutral hydrogen with moderate column density, $N_{\mathrm{H}}^{z} \simeq 10^{21}-10^{22} \mathrm{~cm}^{-2}$, in the majority of the sources (Table 3, Figure 1, middle); (4) possibly a dense Compton-thick absorber in $2021+614$ and $1511+0518$ (Section 4.2). 
Recent multi-wavelength studies also provide evidence that CSO radio sources are embedded in complex gaseous environments. In the IR band the Spitzer data show a variety of IR line properties indicating an enhanced star formation rate in host galaxies of compact radio sources (Willett et al. 2010; Dicken et al. 2012; Guillard et al. 2012). One explanation could be jet-induced star formation in CSOs (Labiano et al. 2008), suggestive of interactions between the jet and the dense environment. Tadhunter et al. (2011) points to a possible selection bias in flux-limited radio samples that impacts general conclusions about the epoch of star formation and triggering of the radio source. However, these IR results are consistent with the CSO source being embedded in a dense environment.

In the radio band, Geréb et al. $(2014,2015)$ show that the properties of the $\mathrm{H}$ I absorption line in the spectra of GPS and compact steep-spectrum sources (CSS; a more evolved stage than the GPS sources) differ substantially from those of the extended radio sources. Based on the correlation between the amount of $\mathrm{H}$ I absorption and the type of radio source $(\sim 55 \%$ $\mathrm{H}_{\mathrm{I}}$ detection rate, and optical depths greater in the compact sources than in the extended sources) the authors argued that the compact radio sources are embedded in a medium that is rich in atomic gas. Moreover, the $\mathrm{H}$ I kinematics in the GPS/ CSS sources (broader and more asymmetric, blueshifted profiles) suggested that the gas is unsettled and may form outflows. They concluded that evidence for interactions between the radio sources and a rich ambient environment is found more frequently in the young/compact sources than in the extended sources.

Finally, studies of the relationship between the X-ray and radio absorbing columns reveal a correlation between the $N_{\mathrm{H}}$ and $N_{\mathrm{H}_{\mathrm{I}}}$ absorption in GPS/CSO sources (Ostorero et al. 2016), suggesting that the $\mathrm{X}$-ray and radio absorbers are co-spatial. This is an important constraint, given the latest radio results from the low-frequency radio observations (Callingham et al. 2015). They seem to rule out the synchrotron self-absorption process and favor free-free absorption as the origin of the radio obscuration in the GPS source, PKS B0008 -42 . This again points to a rich, multi-phase environment impacting the evolution of CSOs.

It is, thus, interesting to interpret our findings on the CSOs' intrinsic column density of neutral hydrogen in the context of the absorption properties of an unbiased population of AGNs. We compare our results with those obtained by Burlon et al. (2011) for their subsample of Sy1.8-2 galaxies from the hard X-ray Swift/BAT survey (note that CSOs are observed at large inclinations to the jet axis, and as such should be compared with Type 2 AGNs). In the sample of CSOs presented in Table 1 (see Figure 5 top) 2 of 15 (13\%) CSOs show evidence for Compton-thick absorption with $N_{\mathrm{H}}^{z} \gtrsim 5 \times 10^{23} \mathrm{~cm}^{-2}$ as compared to $20 \%$ in Sy1.8-2 galaxies in Burlon et al. (2011). Our estimate of a Compton-thick CSO fraction increases to $36 \%$ if we include Compton-thick candidates in this work $(2021+614$ and $1511+0518)$ and in Tengstrand et al. (2009; $1607+26)$.Conversely, nine of $15(60 \%)$ sources have $N_{\mathrm{H}}^{z}<10^{22} \mathrm{~cm}^{-2}(40 \%$ if we exclude $2021+614,1511$ +0518 , and $1607+26)$. Instead, only $7 \%$ of the Sy1.8-2 galaxies have column densities in this range. While the fraction of Compton-thick CSOs seems to be in relatively good agreement with that found in the Sy1.8-2 population of Burlon et al. (2011), especially given the small size of our sample of CSOs, the overabundance of sources with only moderate
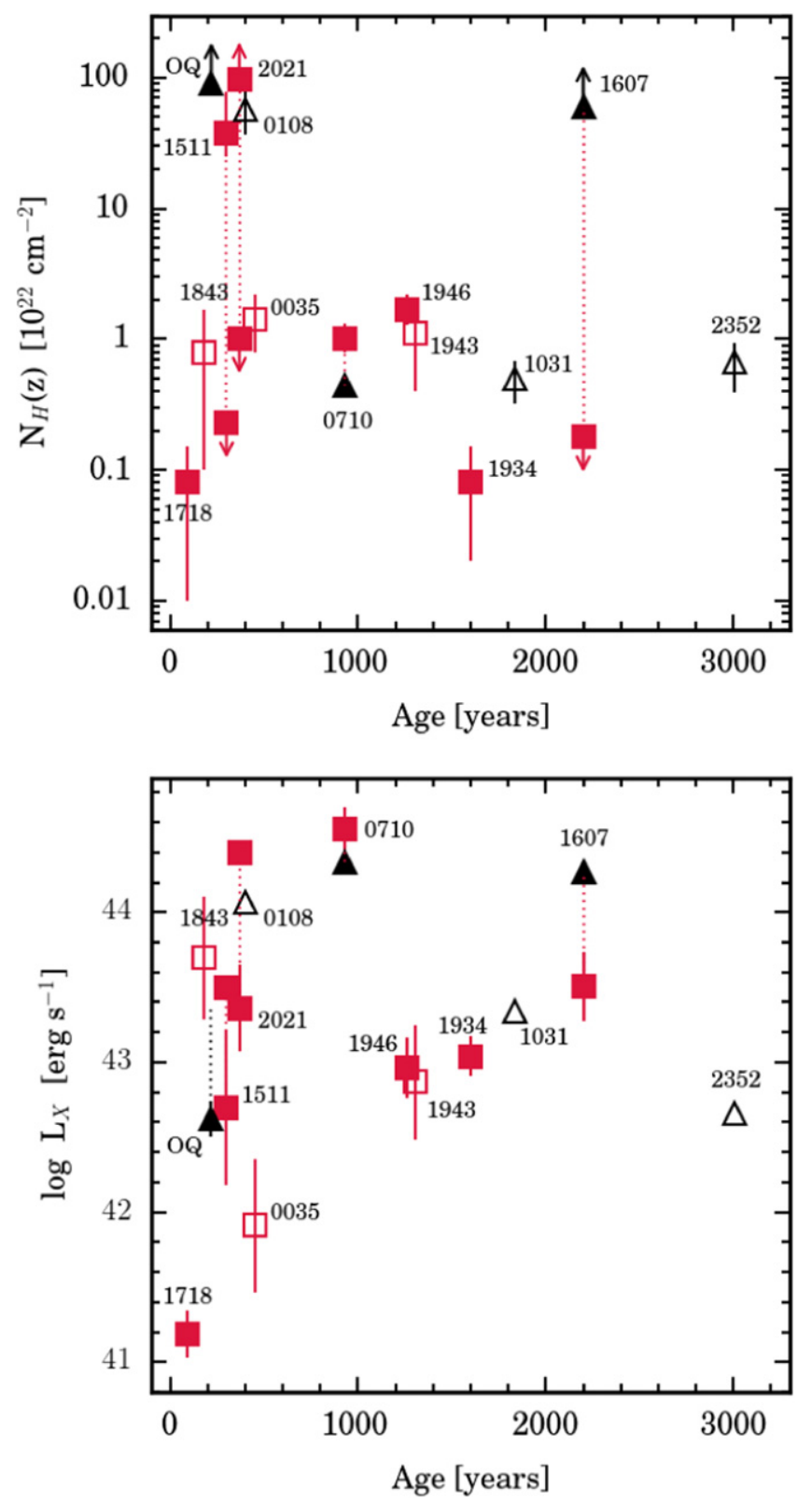

Figure 5. Top: intrinsic X-ray absorbing column versus age based on our Chandra results (squares) and XMM-Newton results (triangles; sources 13-16 in Table 1; filled and open symbols indicate fits with $\Gamma$ free and fixed, respectively; vertical lines connect multiple measurements (or measurements obtained through alternative models as in Section 4.2) available for the same source. Bottom: intrinsic X-ray luminosity (2-10 keV) versus age based on our Chandra results (squares, $K$-corrected) and XMM-Newton results (triangles; sources 6, 10, 13-16 in Table 1). Symbols as in the top panel; note the large scatter within the youngest sources.

intrinsic obscuration seems puzzling. Hardcastle et al. (2009) pointed out that the narrow-line radio galaxies (NLRG), considered to be the more evolved analogues of CSOs, have $N_{\mathrm{H}}^{z} \gtrsim 3 \times 10^{22} \mathrm{~cm}^{-2}$ associated with the absorption of the nuclear emission by a molecular torus. Consequently, the large fraction of CSOs with hydrogen column densities lower than those typically found in NLRGs may indicate-echoing the conclusions of Ostorero et al. (2016) — that the CSO X-ray obscuration is caused by the gas located on much larger scales than molecular tori. 


\subsection{X-Ray Continuum of CSO}

The 2-10 keV X-ray luminosities of our sources cover more than three orders of magnitude (Figure 5, bottom), $L_{\mathrm{X}} \simeq 2 \times 10^{41}-6 \times 10^{44} \mathrm{erg} \mathrm{s}^{-1}$, typical for the $z<1$ AGNs (e.g Akylas et al. 2006; Winter et al. 2009; Fukazawa et al. 2011; Jia et al. 2013; Aird et al. 2015). Interestingly, we observed that the oldest $\operatorname{CSOs}(t>1000 \mathrm{yr})$ tend to be relatively bright in X-rays, with luminosities $L_{\mathrm{X}}>10^{43} \mathrm{erg} \mathrm{s}^{-1}$, while the youngest CSOs can be found at any X-ray luminosity covered by the sample.

We found hard X-ray spectra with a photon index $\Gamma \sim 1.4$ 1.7 in five (out of seven) sources where we were able to model the photon index. Among non-blazar AGNs, this range of photon indices is often seen in radio-quiet AGNs, although it appears to be most compatible with the average photon index of the non-blazar radio-loud AGNs (Reeves \& Turner 2000; Kelly et al. 2008; Sobolewska et al. 2009, 2011; but see Young et al. 2009). The difference between the average X-ray spectral slopes in radio-loud and radio-quiet AGN populations may result from either a dilution of the $\mathrm{X}$-ray coronal emission or a non-negligible contribution from the non-thermal emission of jets (Belsole et al. 2006). It is, thus, likely that in the particular case of CSOs the X-ray jets and/or lobes contribute to the observed X-ray radiation, as suggested by Stawarz et al. (2008) and Ostorero et al. (2010). The photon indices we observed would then be consistent with a non-thermal scenario in which low-energy photons are upscattered by the inverse Compton process to X-ray energies by electrons with a power-law spectral energy distribution with a rather standard spectral index, $p \sim 2$.

Thus, the shape of the absorbed power-law X-ray continuum that we derived for the CSOs in our sample is consistent with either the nuclear (X-ray corona) emission absorbed on the torus scale $(\lesssim \mathrm{pc})$ or an extended (X-ray jets/lobes) emission absorbed on the scale of the host galaxy $(\sim \mathrm{kpc})$. We note that the results described in Section 5.2, along with that of Ostorero et al. (2016), favor the latter possibility. Instead, high intrinsic luminosities, exceeding $10^{44} \mathrm{erg} \mathrm{s}^{-1}$, derived for several CSOs (Figure 5, bottom panel), together with the likely detection of the reprocessed/scattered nuclear light in $2021+614,1511$ +0518 (Section 4.2), and 0710+439 (Section 4.1), may indicate the dominant AGN contribution to the observed $\mathrm{X}$-ray emission of young radio sources.

Our results do not appear to favor thermal emission of the diffuse ISM plasma as the origin of the CSO X-ray continuum, unless the soft X-rays and the He-like iron emission in 0710 +439 are interpreted as a sole example in which the ISM is heated by the expanding radio structure (Section 4.1, Figure 4, Model E).

\section{SUMMARY AND CONCLUSIONS}

We examined the X-ray properties of 16 young radio sources with Chandra and observed a variety of interesting characteristics, which we summarize below.

1. We found that our CSO sample covers a wide range in $\mathrm{X}$-ray luminosity, $L_{2-10 \mathrm{keV}} \sim 10^{41}-10^{45} \mathrm{erg} \mathrm{s}^{-1}$, and that the majority of sources contain a modest amount of intrinsic absorption $\left(N_{\mathrm{H}} \simeq 10^{21}-10^{22} \mathrm{~cm}^{-2}\right)$.

2. We obtained the highest quality $X$-ray spectrum of the CSO source to date in our long Chandra observation of $0710+439$ and found an ionized iron emission line,
$E_{\text {rest }}=(6.62 \pm 0.04) \mathrm{keV}$. However, we were not able to determine the origin of the line, because it could be related to the emission from the hot ionized accretion disk illuminated by an external source, from a distant photoionized medium, or from a diffuse hot thermal plasma within the nuclear region. Depending on the model, we derived an $\mathrm{EW}$ of the line ranging from $154_{-58}^{+65} \mathrm{eV}$ (Model B) to $1.40_{-0.79}^{+0.97} \mathrm{keV}$ (Model F).

3. We found a hard photon index of $\Gamma=0.8_{-0.2}^{+0.3}$ in 2021 +614 and $\Gamma=1.0 \pm 0.2$ in $1511+0518$ consistent with either a Compton-thick absorber or non-thermal emission from compact radio lobes.

4. We discovered that the $2-10 \mathrm{keV} X$-ray flux decreased by an order of magnitude since the 2008 XMM-Newton observation of $1607+26$. Future monitoring of this source is needed in order to characterize and understand the nature of the variability.

5. We examined X-ray images of the CSOs with the highest angular resolution and detected extended X-ray emission in 1718-649, which needs to be studied with deeper $\mathrm{X}$-ray observations in the future.

We found that short ( $\sim 5 \mathrm{ks})$ X-ray observations with Chandra have proven to be effective in detecting the sources of CSOs and deriving their basic X-ray properties. With our Chandra program, we observed six CSOs for the first time in $\mathrm{X}$-rays, and we increased the size of the known sample of CSOs with measured redshift, kinematic age, and X-ray information by $\sim 68 \%$. The CSO X-ray population can be further extended through snapshot Chandra and/or XMMNewton observations of seven sources whose kinematic ages have been derived recently (An \& Baan 2012, Figure 1). However, it is apparent that deeper exposures are needed in order to study details of the CSOs' environment (e.g., the amount of intrinsic absorption, the gaseous media co-spatial with the radio sources) and the origin of the CSOs' intrinsic $\mathrm{X}$-ray emission (diffused thermal versus nuclear emission, evidence for reprocessing of the nuclear X-rays by circumnuclear matter, a thermal versus non-thermal electron population generating the high-energy spectra of CSOs, long-term $\mathrm{X}$-ray variability). Deep X-ray observations are also necessary in order to place critical constraints on current theoretical models for the earliest stage of the evolution of radio sources.

We thank the anonymous referee for detailed comments. This research is funded in part by National Aeronautics and Space Administration contract NAS8-03060. Partial support for this work was provided by the NASA grants GO1-12145X, GO4-15099X, NNX10AO60G. G.M. acknowledges the financial support from the UnivEarthS Labex program of Sorbonne Paris Cité (ANR10LABX0023 and ANR11IDEX000502). L. O. acknowledges support from the INFN grant INDARK, the grant PRIN 2012 "Fisica Astroparticellare Teorica" of the Italian Ministry of University and Research, and the "Strategic Research Grant: Origin and Detection of Galactic and Extragalactic Cosmic Rays" funded by the University of Torino and Compagnia di San Paolo. Ł.S. was supported by Polish NSC grant DEC-2012/04/A/ST9/00083. This research has made use of data obtained by the Chandra X-ray Observatory, and software provided by Chandra X-ray Center (CXC) in the application packages CIAO, ChIPS, and Sherpa. 


\section{REFERENCES}

Aird, J., Coil, A. L., Georgakakis, A., et al. 2015, MNRAS, 451, 1892

Akylas, A., Georgantopoulos, I., Georgakakis, A., Kitsionas, S., \& Hatziminaoglou, E. 2006, A\&A, 459, 693

An, T., \& Baan, W. A. 2012, ApJ, 760, 77

An, T., Wu, F., Yang, J., et al. 2012, ApJS, 198, 5

Astropy Collaboration, Robitaille, T. P., Tollerud, E. J., et al. 2013, A\&A, 558, A33

Begelman, M. C. 1996, Proceedings of the Greenbank Workshop, Cygnus AStudy of a Radio Galaxy, ed. C. L. Carilli \& D. E. Harris (Cambridge: Cambridge Univ. Press), 209

Begelman, M. C., Blandford, R. D., \& Rees, M. J. 1984, RvMP, 56, 255

Begelman, M. C., \& Cioffi, D. F. 1989, ApJL, 345, L21

Belsole, E., Worrall, D. M., \& Hardcastle, M. J. 2006, MNRAS, 366, 339

Best, P. N., Kaiser, C. R., Heckman, T. M., \& Kauffmann, G. 2006, MNRAS, 368, L67

Bianchi, S., \& Matt, G. 2002, A\&A, 387, 76

Bianchi, S., Matt, G., Balestra, I., Guainazzi, M., \& Perola, G. C. 2004, A\&A, 422, 65

Bicknell, G. V., Dopita, M. A., \& O’Dea, C. P. O. 1997, ApJ, 485, 112

Burlon, D., Ajello, M., Greiner, J., et al. 2011, ApJ, 728, 58

Callingham, J. R., Gaensler, B. M., Ekers, R. D., et al. 2015, ApJ, 809, 168

Carilli, C. L., Perley, R. A., \& Dreher, J. H. 1988, ApJL, 334, L73

Carvalho, J. C. 1994, A\&A, 292, 392

Cash, W. 1979, ApJ, 228, 939

Comastri, A. 2004, Supermassive Black Holes in the Distant Universe, Vol. 308 (Dordrecht: Kluwer), 245

Croton, D. J., Springel, V., White, S. D. M., et al. 2006, MNRAS, 365, 11

Czerny, B., Siemiginowska, A., Janiuk, A., Nikiel-Wroczyński, B., \& Stawarz, Ł. 2009, ApJ, 698, 840

De Young, D. S. 1993, ApJ, 402, 95

De Young, D. S. 1997, ApJL, 490, L55

Dicken, D., Tadhunter, C., Axon, D., et al. 2012, ApJ, 745, 172

Done, C., Sobolewska, M. A., Gierliński, M., \& Schurch, N. J. 2007, MNRAS, 374, L15

Dutson, K. L., Edge, A. C., Hinton, J. A., et al. 2014, MNRAS, 442, 2048

Fanti, C., Fanti, R., Dallacasa, D., et al. 1995, A\&A, 302, 317

Freeman, P., Doe, S., \& Siemiginowska, A. 2001, Proc. SPIE, 4477, 76

Fruscione, A., McDowell, J. C., Allen, G. E., et al. 2006, Proc. SPIE, 6270, 60

Fukazawa, Y., Hiragi, K., Mizuno, M., et al. 2011, ApJ, 727, 19

García-Burillo, S., Combes, F., Neri, R., et al. 2007, A\&A, 468, L71

George, I. M., \& Fabian, A. C. 1991, MNRAS, 249, 352

Geréb, K., Maccagni, F. M., Morganti, R., \& Oosterloo, T. A. 2015, A\&A, 575, A44

Geréb, K., Morganti, R., \& Oosterloo, T. A. 2014, A\&A, 569, A35

Giroletti, M., \& Polatidis, A. 2009, AN, 330, 193

Gopal-Krishna, \& Wiita, P. J. 1991, ApJ, 373, 325

Guainazzi, M., Siemiginowska, A., Rodriguez-Pascual, P., \& Stanghellini, C. 2004, A\&A, 421, 461

Guainazzi, M., Siemiginowska, A., Stanghellini, C., et al. 2006, A\&A, 446, 87

Gugliucci, N. E., Taylor, G. B., Peck, A. B., \& Giroletti, M. 2005, ApJ, 622, 136

Guillard, P., Ogle, P. M., Emonts, B. H. C., et al. 2012, ApJ, 747, 95

Hardcastle, M. J., Evans, D. A., \& Croston, J. H. 2009, MNRAS, 396, 1929

Hardcastle, M. J., Massaro, F., Harris, D. E., et al. 2012, MNRAS, 424, 1774

Heinz, S., Reynolds, C. S., \& Begelman, M. C. 1998, ApJ, 501, 126

Hinshaw, G., Larson, D., Komatsu, E., et al. 2013, ApJS, 208, 19

Holt, J., Tadhunter, C., \& Morganti, R. 2003, NewAR, 47, 261

Holt, J., Tadhunter, C. N., \& Morganti, R. 2008, MNRAS, 387, 639

Iwasawa, K., Mainieri, V., Brusa, M., et al. 2012, A\&A, 537, A86

Jia, J., Ptak, A., Heckman, T., \& Zakamska, N. L. 2013, ApJ, 777, 27

Jiang, D. R., Dallacasa, D., Schilizzi, R. T., et al. 1996, A\&A, 312, 380

Kashyap, V. L., van Dyk, D. A., Connors, A., et al. 2010, ApJ, 719, 900

Kataoka, J., Stawarz, Ł., Cheung, C. C., et al. 2010, ApJ, 715, 554

Kelly, B. C., Bechtold, J., Trump, J. R., Vestergaard, M., \& Siemiginowska, A. 2008, ApJS, 176, 355

King, I. 1962, AJ, 67, 471

Kraft, R. P., Forman, W., Jones, C., et al. 2000, ApJL, 531, L9

Krolik, J. H., \& Kallman, T. R. 1987, ApJL, 320, L5

Kunert-Bajraszewska, M., Gawroński, M. P., Labiano, A., \& Siemiginowska, A. 2010, MNRAS, 408, 2261

Kunert-Bajraszewska, M., Labiano, A., Siemiginowska, A., \& Guainazzi, M. 2014, MNRAS, 437, 3063
Kunert-Bajraszewska, M., Labiano, A., Siemiginowska, A., Guainazzi, M., \& Gawroński, M. 2015, in IAU Symp. 313, Extragalactic Jets from Every Angle, ed. F. Massaro, C. C. Cheung, E. Lopez, \& A. Siemiginowska (Cambridge: Cambridge Univ. Press), 242

Kuraszkiewicz, J., Wilkes, B. J., Schmidt, G., et al. 2009, ApJ, 692, 1143

Labiano, A., O'Dea, C. P., Barthel, P. D., de Vries, W. H., \& Baum, S. A. 2008, A\&A, 477, 491

Luo, W.-F., Yang, J., Cui, L., Liu, X., \& Shen, Z.-Q. 2007, ChJAA, 7, 611

Matt, G., Fabian, A. C., \& Ross, R. R. 1993, MNRAS, 262, 179

Matt, G., Guainazzi, M., \& Maiolino, R. 2003, MNRAS, 342, 422

Migliori, G., Grandi, P., Torresi, E., et al. 2011, A\&A, 533, A72

Migliori, G., Siemiginowska, A., \& Celotti, A. 2012, ApJ, 749, 107

Migliori, G., Siemiginowska, A., Kelly, B. C., et al. 2014, ApJ, 780, 165

Mingo, B., Hardcastle, M. J., Croston, J. H., et al. 2011, ApJ, 731, 21

Morganti, R. 2008, in Extragalactic Jets: Theory and Observation from Radio to Gamma Ray, Vol. 386, ed. T. A. Rector, \& D. S. De Young (San Francisco, CA: ASP), 210

Morganti, R., Fogasy, J., Paragi, Z., Oosterloo, T., \& Orienti, M. 2013, Sci, 341,1082

Morganti, R., Tadhunter, C. N., Oosterloo, T. A., et al. 2003, PASA, 20, 129

Müller, C., Kadler, M., Ojha, R., et al. 2014, A\&A, 562, A4

Müller, C., Krauß, F., Dauser, T., et al. 2015, A\&A, 574, A117

Nagai, H., Inoue, M., Asada, K., Kameno, S., \& Doi, A. 2006, ApJ, 648, 148

Nandra, K., O’Neill, P. M., George, I. M., \& Reeves, J. N. 2007, MNRAS, 382, 194

Nelder, J. A., \& Mead, R. 1965, CompJ, 7, 308

O’Dea, C. P. 1998, PASP, 110, 493

O’Dea, C. P., \& Baum, S. A. 1997, AJ, 113, 148

O’Dea, C. P., De Vries, W. H., Worrall, D. M., Baum, S. A., \& Koekemoer, A. 2000, AJ, 119, 478

Ostorero, L., Moderski, R., Stawarz, Ł., et al. 2010, ApJ, 715, 1071

Ostorero, L., Morganti, R., Diaferio, A., et al. 2016, AN, 337, 148

Owsianik, I., \& Conway, J. E. 1998, A\&A, 337, 69

Patrick, A. R., Reeves, J. N., Porquet, D., et al. 2012, MNRAS, 426, 2522

Perucho, M. 2015, arXiv:1507.03355

Polatidis, A. G., \& Conway, J. E. 2003, PASA, 20, 69

Porquet, D., Reeves, J. N., O’Brien, P., \& Brinkmann, W. 2004, A\&A, 422, 85

Protassov, R., van Dyk, D. A., Connors, A., Kashyap, V. L., \& Siemiginowska, A. 2002, ApJ, 571, 545

Readhead, A. C. S., Taylor, G. B., Pearson, T. J., \& Wilkinson, P. N. 1996a, ApJ, 460, 634

Readhead, A. C. S., Taylor, G. B., Xu, W., et al. 1996b, ApJ, 460, 612

Reeves, J. N., \& Turner, M. J. L. 2000, MNRAS, 316, 234

Refsdal, B., et al. 2009, in Proc. VIII Python in Science Conf. (SciPy 2009), ed. G. Varoquaux, S. van der Walt, \& J. Millman, 51 http://conference. scipy.org/proceedings/SciPy2009/paper_8

Reynolds, C. S., \& Begelman, M. C. 1997, ApJL, 487, L135

Reynolds, C. S., Heinz, S., \& Begelman, M. C. 2001, ApJL, 549, L179

Risaliti, G., Elvis, M., Bianchi, S., \& Matt, G. 2010, MNRAS, 406, L20

Risaliti, G., Elvis, M., Fabbiano, G., Baldi, A., \& Zezas, A. 2005, ApJL, 623, L93

Risaliti, G., Nardini, E., Salvati, M., et al. 2011, MNRAS, 410, 1027

Risaliti, G., Woltjer, L., \& Salvati, M. 2003, A\&A, 401, 895

Różańska, A., Dumont, A.-M., Czerny, B., \& Collin, S. 2002, MNRAS, 332, 799

Scheuer, P. A. G. 1974, MNRAS, 166, 513

Shulevski, A., Morganti, R., Oosterloo, T., \& Struve, C. 2012, A\&A, 545, A91

Siemiginowska, A. 2009, AN, 330, 264

Siemiginowska, A., LaMassa, S., Aldcroft, T. L., Bechtold, J., \& Elvis, M. 2008, ApJ, 684, 811

Siemiginowska, A., Stawarz, Ł., Cheung, C. C., et al. 2012, ApJ, 750, 124

Silk, J., \& Rees, M. J. 1998, A\&A, 331, L1

Smith, R. K., Brickhouse, N. S., Liedahl, D. A., \& Raymond, J. C. 2001, ApJL, 556, L91

Sobolewska, M. A., Gierliński, M., \& Siemiginowska, A. 2009, MNRAS, 394, 1640

Sobolewska, M. A., Siemiginowska, A., \& Gierliński, M. 2011, MNRAS, 413, 2259

Stawarz, Ł., Ostorero, L., Begelman, M. C., et al. 2008, ApJ, 680, 911

Tadhunter, C., Holt, J., González Delgado, R., et al. 2011, MNRAS, 412, 960

Tadhunter, C., Morganti, R., Rose, M., Oonk, J. B. R., \& Oosterloo, T. 2014, Natur, 511, 440

Taylor, G. B., Marr, J. M., Pearson, T. J., \& Readhead, A. C. S. 2000, ApJ, 541,112 
Tengstrand, O., Guainazzi, M., Siemiginowska, A., et al. 2009, A\&A, 501, 89 Tingay, S. J., Macquart, J.-P., Collier, J. D., et al. 2015, AJ, 149, 74

Tsujimoto, M., Guainazzi, M., Plucinsky, P. P., et al. 2011, A\&A, 525, A25 van Breugel, W., Miley, G., \& Heckman, T. 1984, AJ, 89, 5

Vink, J., Snellen, I., Mack, K.-H., \& Schilizzi, R. 2006, MNRAS, 367, 928

Wagner, A. Y., \& Bicknell, G. V. 2011, ApJ, 728, 29

Watson, M. G., Schröder, A. C., Fyfe, D., et al. 2009, A\&A, 493, 339
Willett, K. W., Stocke, J. T., Darling, J., \& Perlman, E. S. 2010, ApJ, 713, 1393

Winter, L. M., Mushotzky, R. F., Reynolds, C. S., \& Tueller, J. 2009, ApJ, 690, 1322

Young, M., Elvis, M., \& Risaliti, G. 2009, ApJS, 183, 17

Zhou, X. L., Zhao, Y. H., \& Soria, R. 2011, MNRAS, 413, L61

Zycki, P. T., \& Czerny, B. 1994, MNRAS, 266, 653 